\title{
Effects of Disorder on the Exchange Coupling in (Ga,Mn)As Diluted Magnetic Semiconductors
}

\author{
Antônio J. R. da Silva, ${ }^{1}$ A. Fazzio, ${ }^{1}$ Raimundo R. dos Santos, ${ }^{2}$ and Luiz E. Oliveira ${ }^{3}$ \\ ${ }^{1}$ Instituto de Física, Universidade de São Paulo, CP 66318, 05315-970, São Paulo SP, Brazil \\ ${ }^{2}$ Instituto de Física, Universidade Federal do Rio de Janeiro, CP 68528, 21945-970 Rio de Janeiro RJ, Brazil \\ ${ }^{3}$ Instituto de Física, Unicamp, CP 6165, 13083-970 Campinas SP, Brazil
}

Received on 8 December, 2005

\begin{abstract}
A theoretical study of the effects of disorder on the Mn-Mn exchange interactions for $\mathrm{Ga}_{1-x} \mathrm{Mn}_{x} \mathrm{As}$ diluted magnetic semiconductors is presented. The disorder is intrinsically considered in the calculations, which are performed using an $a b$ initio total energy density-functional approach, for a 128 atoms supercell, and by considering a variety of configurations with 2,3 and $4 \mathrm{Mn}$ atoms. Results are obtained for the effective $\mathrm{J}_{n}^{M n-M n}$, from first $(n=1)$ all the way up to sixth $(n=6)$ neighbors via a Heisenberg Hamiltonian used to map the magnetic excitations from $a b$ initio total energy calculations. One then obtains a clear dependence in the magnitudes of the $\mathrm{J}_{n}^{M n-M n}$ with the Mn concentration $x$. Moreover, we show that, in the case of fixed Mn concentration, configurational disorder and/or clustering effects lead to large dispersions in the Mn-Mn exchange interactions. Also, calculations for the ground-state total energies for several configurations suggest that a proper consideration of disorder is needed when one is interested in treating temperature and annealing effects.
\end{abstract}

Keywords: Disorder; Exchange coupling; Diluted magnetic semiconductors

In the past few years a lot of attention has been given to the study of diluted magnetic semiconductors (DMS), due to possible applications in new devices by manipulating both the spin and the charge of the carriers in semiconductor systems. This was even more so after the successful growth of ferromagnetic (Ga,Mn)As alloys [1], in which the critical temperature and hole concentration (cf. Fig. 1), as a function of Mn composition, are very much dependent on the details of growth conditions [2-14]. From the theoretical point of view, several calculations [15-24] have been performed to extract exchange couplings ( $\left.\mathrm{J}^{\prime} \mathrm{s}\right)$ for specific clusters containing $\mathrm{Mn}$ ions, with overall results suggesting a tendency of a decrease in $|J|$ when more $\mathrm{Mn}$ atoms are added to nearby sites, and a considerable scatter in the values of the exchange couplings. Here we stress that the use of non-full potential muffin-tin-style approaches is not adequate to treat the electronic structure of covalent semiconductor systems such as (Ga,Mn)As DMS, and that disorder quite certainly is not realistically treated by simple effective-medium approaches such as the virtual crystal approximation (VCA) or CPA.

We have performed total energy calculations within the density-functional theory (DFT) and the generalized-gradient approximation (GGA) for the exchange-correlation potential, with the electron-ion interactions described using ultrasoft pseudopotentials [25], and plane wave expansion up to 230 $\mathrm{eV}$ as implemented in the VASP code [26]. For a 128 atoms supercell, we consider a variety of configurations with 2,3 and $4 \mathrm{Mn}$ atoms, corresponding to concentrations of $3.1 \%, 4.7 \%$, and $6.3 \%$, respectively. We have considered typical configurations, as generated through the Special Quasi-random Structures (SQS) algorithm [27]. Results for the effective $\mathrm{J}_{n}^{M n-M n}$ exchange couplings, from first $(n=1)$ all the way up to sixth $(n=6)$ neighbors, are obtained through a Heisenberg Hamiltonian used to map the magnetic excitations from ab initio total energy calculations. Details of the calculation may be found elsewhere [28-30]. In the case of two Mn substitutional atoms in the 128-site supercell (Mn concentration of $x=3.1 \%$ ), we have considered all configurations correspond- ing to all inequivalent positions within the supercell, i.e., MnMn distances varying from $4.1 \AA$ up to $11.5 \AA$, and total energy results yield a Mn-Mn ferromagnetic ground state in all cases, with the resulting Mn-Mn ferromagnetic effective coupling in $\mathrm{Ga}_{1-x} \mathrm{Mn}_{x}$ As essentially intermediated by the antiferromagnetic coupling of each $\mathrm{Mn}$ spin to the quasi-localized holes [28-30] (cf. open dots in Fig. 2). Also, it is clear that the observed non-monotonic behavior of $J_{n}$ is essentially due to the anisotropic character of the effective interaction. Moreover, $\left|J_{n}\right|$ essentially decreases with Mn-Mn separation and vanishes above $\sim 11.5 \AA$.

For three Mn atoms in a supercell with 128 sites, we have performed calculations for 10 different disorder configurations. Fig. 3 (a) shows an illustrative SQS configuration, in which the $3 \mathrm{Mn}$ atoms are somewhat clustered together. Fig. 2 shows the exchange coupling $J_{n}$ as a function of $n$ for the 10 SQS realizations of disorder (three Mn atoms, $x=4.7 \%$ ); for comparison, we show the results for $x=3.1 \%$ in the same figure. Note that the overall trend of $J_{n}$ with $n$, observed in the case of two Mn spins, is maintained, and the non-monotonic behavior is still due to the fact that the exchange coupling depends not only on the distance between the pair of Mn atoms, but also on their relative direction with respect to the bonds of the host GaAs. It is important to mention that the present 128-atom supercell total energy results for the ferro- and antiferromagnetic states are in overall agreement with the 64-atom supercell total energy results of Mahadevan et al [31,32].

We have also performed calculations for non-SQS configurations in which two $\mathrm{Mn}$ atoms are first neighbors, and a third $\mathrm{Mn}$ atom is placed in positions corresponding to fifth-, third, and first-neighbor of the pair: we found that $J_{1}=-20.8$ $\mathrm{meV},-17.3 \mathrm{meV}$, and $-8.1 \mathrm{meV}$, respectively. Thus, clustering tends to weaken the magnitude of the nearest-neighbor coupling. One may attribute this behavior as most likely resulting from the Coulomb repulsion between the holes, which leads to their delocalization as the $\mathrm{Mn}$ atoms group together, being therefore detrimental of their role as mediators of ferromagnetism. If, on the one hand, clustering tends to decrease 

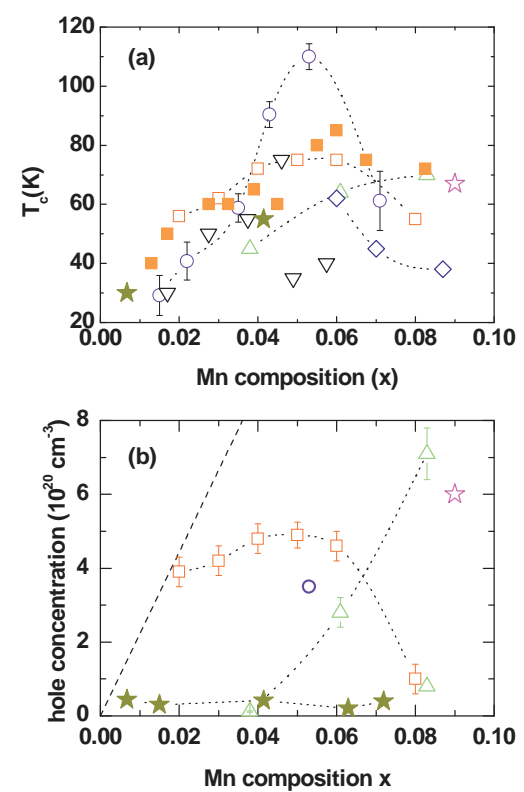

FIG. 1: (Color online) Experimental critical temperature (a) and hole concentration (b) as functions of Mn composition in $\mathrm{Ga}_{1-x} \mathrm{Mn}_{x} \mathrm{As}$ alloys: diamonds are data from van Esch et al [2], circles from Matsukura et al [3] and Ohno et al [4], up triangles from Potashnik et al [5] and Seong et al [7], open squares from Edmonds et al [6], down triangles from Asklund et al [8], open stars from from Yu et al $[9,10]$, full squares from Potashnik et al [11], and full stars from Moriya and Munekata [12]. Dotted lines through data points are guides to the eye. The dashed line in (b) corresponds to a hole concentration equal to that of the Mn sites.

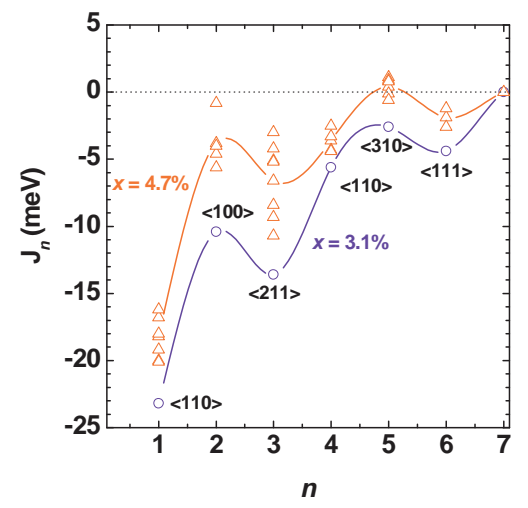

FIG. 2: (Color online) The $n$ th-nearest-neighbor exchange coupling for $x=3.1 \%$ and $x=4.7 \%$. Full curves are guides to the eye (for $x=$ $4.7 \%$ the full line goes through average values of $J_{n}$ ). The direction $<h k l>$ of each $n$-th neighbor pair is also shown.

the magnitude of the nearest-neighbor exchange, on the other hand it leads to the energetically most stable configuration; this is in agreement with results from recent calculations restricted to pairs of transition metals [33]. Fig. 3(b) shows the net magnetization $m(\mathbf{r}) \equiv \rho_{\uparrow}(\mathbf{r})-\rho_{\downarrow}(\mathbf{r})$, where $\rho_{\sigma}$ is the total charge density in the $\sigma$-polarized channel, for three $\mathrm{Mn}$ atoms with all spins aligned, for the configuration depicted in Fig. 3(a). By performing calculations in which there is only one flipped spin, one obtains a similar behavior for $m(\mathbf{r})$ as

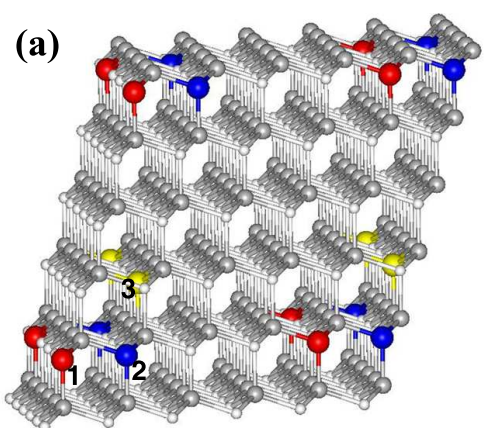

(b)

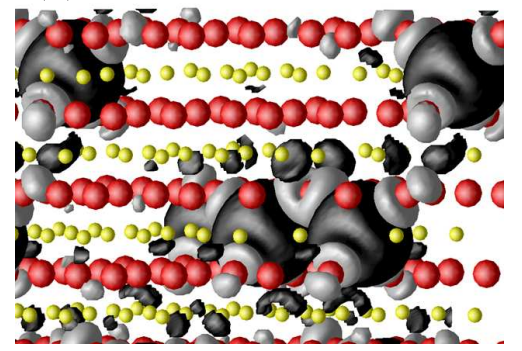

FIG. 3: (Color online) (a) A pictorial view of a possible realization of disorder for three $\mathrm{Mn}$ atoms in a 128 -site supercell $(x=4.7 \%)$. Ga sites are represented by the smaller spheres, As sites by the middlesized ones, and Mn atoms by the largest ones. For clarity, supercells are repeated along the different cartesian directions. The three nonequivalent $\mathrm{Mn}$ atoms are shown as different shades of gray (blue, red, and yellow in the color version); (b) Isosurfaces for the net local magnetization $m(\mathbf{r})$ (see text for definition) in the case of three $\mathrm{Mn}_{\mathrm{Ga}}$ defects [for the configuration depicted in (a)], with all spins aligned. The black surface corresponds to a value of $+0.005 e / \AA^{3}$, and the grey surface to $-0.005 e / \AA^{3}$, with $e$ being the electron charge. The smaller-yellow (larger-red) spheres denote the Ga (As) atoms.

in the case of one [28] and two Mn impurities [29] in a supercell: near each $\mathrm{Mn}$ atom the local magnetization has a $d_{\sigma}-$ like character, whereas close to the As neighbors, the character changes to $p_{\bar{\sigma}}$-like, where $\sigma=(\uparrow$ or $\downarrow)$ and $\bar{\sigma}=(\downarrow$ or $\uparrow)$. Also, $m(\mathbf{r})$ has a rather localized character.

For four Mn atoms, we have considered only two disorder configurations, chosen according to the SQS algorithm. One sees (see Fig. 4) that the overall tendency of $\left|J_{n}\right|$ is to decrease in magnitude as $n$ is increased, in a pattern similar to that for smaller concentrations, though the dispersion cannot be properly assessed due to the scarcity of data. We also note that, as in the case of three $\mathrm{Mn}$ atoms, calculations with a nonSQS configuration with the four Mn atoms clustered together indicate that clustering decreases the magnitude of the firstneighbor $J_{1}$ exchange coupling: $J_{1}=-6.5 \mathrm{meV}$ in this case, which should be compared with the $-12.6 \mathrm{meV}$ and -13.0 $\mathrm{meV}$ values previously obtained. From Fig. 4, we see that, in most cases, the magnitudes of the exchange couplings decrease as the concentration of $\mathrm{Mn}$ atoms is increased, a behavior that may be understood from the fact that the effective Mn-Mn interaction is hole-mediated, thus sensitive to the hole density. Further, this decrease may be quite significant; for instance, the magnitude of the average $J_{1}$ decreases by the or- 


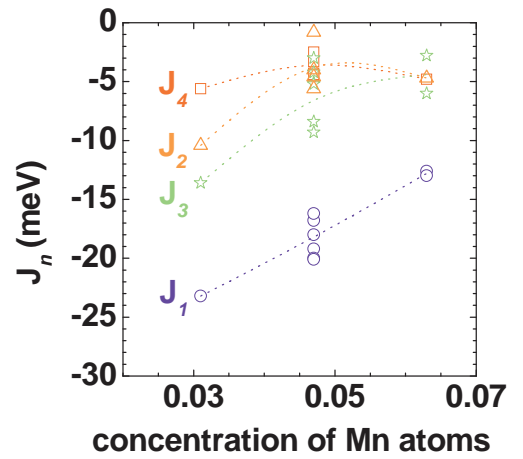

FIG. 4: (Color online)Dependence of $J_{1}, J_{2}, J_{3}$, and $J_{4}$ with the concentration of Mn atoms. For $x=3.1 \%, J_{n}$ is unique for a given $n$. Values for the SQS configurations are shown as empty symbols, and dotted curves are guides to the eye through the average values of $J_{n}$.

der of $50 \%$ when one roughly doubles the concentration from $3.1 \%$. This overall decrease with $x$ can be taken as numerical evidence that a steady increase in the concentration of $\mathrm{Mn}$ atoms is not sufficient to rise the critical temperature, since the exchange couplings will eventually be weakened. Clearly other effects may be playing important roles. Nonetheless, one expects that the trends unveiled here are indicative of the actual experimental situation.

As mentioned before, several theoretical works have previously examined the dependence of the exchange couplings with the Mn-Mn separation or with the Mn concentration [1524] Some predict an alternating sign for the exchange coupling, but these predictions should be taken with extreme care, since these theoretical calculations are based on nonfull-potential muffin-tin-type potentials which are not reliable to treat the electronic structure of covalent semiconductor systems such as (Ga,Mn)As DMS. Also, disorder quite certainly is not adequately taken into account within simple effectivemedium approaches such as VCA or CPA, as fluctuations in the $\mathrm{Mn}$ positions essentially lead to variations in the $\mathrm{Mn}-\mathrm{Mn}$ exchange-coupling parameters, as apparent from Fig. 4.

In conclusion, we have performed $a b$ initio total energy density-functional calculations for substitutional $\mathrm{Mn}$ atoms in a 128 atoms supercell, corresponding to concentrations of $3.1 \%, 4.7 \%$, and $6.3 \%$, respectively. In this way, we have treated the host and the Mn impurities on equal footing. While the relation between the densities of holes and of $\mathrm{Mn}$ atoms is one of the yet unsolved issues in the context of DMS, here we have assumed that each Mn atom provides one hole; since our results relate to general trends, they may be carried over to the actual experimental situation of only a fraction of $\mathrm{Mn}$ atoms contributing with holes. It is also interesting to note that the cut-off of $11.5 \AA$ (which would correspond to $x \simeq 0.042$ ) imposed on the range of Mn-Mn exchange couplings would appear to be in direct contradiction with experimental data by Edmonds et al [6], according to which ferromagnetism is seen for dopings as low as $\sim 0.015$ (where one would have essentially no compensation). Since the site percolation threshold [34] for FCC lattices is 0.20 , for the Ga FCC sublattice in (Ga,Mn)As, the concentration cut-off for ferromagnetic order would be of the order of $0.20 \times 0.042=0.0084$, i.e., $x \simeq 0.84 \%$, indicating that there is no contradiction with the measurements of Edmonds et al [6]. We should also stress that the present results corroborate that the Mn-Mn ferromagnetic effective coupling in $\mathrm{Ga}_{1-x} \mathrm{Mn}_{x}$ As is intermediated by localized holes leading to an antiferromagnetic (non-RKKY) coupling of each Mn spin, as previously noted [28,29], and recently confirmed experimentally [35]. Therefore, the inescapable conclusion is that the main feature of a conventional free-electron-like or perturbative RKKY interaction should be ruled out $[36,37]$ in the case of $\mathrm{Ga}_{1-x} \mathrm{Mn}_{x} \mathrm{As}$.

\section{Acknowledgments}

Partial financial support by the Brazilian Agencies CNPq, CENAPAD-Campinas, Rede Nacional de Materiais Nanoestruturados/CNPq, FAPESP, FAPERJ, and Millenium Institute for Nanosciences/MCT is gratefully acknowledged.
[1] H. Ohno, A. Shen, F. Matsukura, A. Oiwa, A. Endo, S. Katsumoto, and Y. Iye, Appl. Phys. Lett. 69, 363 (1996).

[2] A. Van Esch, L. Van Bockstal, J. De Boeck, G. Verbanck, A. S. van Steenbergen, P. J. Wellmann, B. Grietens, R. Bogaerts, F. Herlach, and G. Borghs, Phys. Rev. B 56, 13103 (1997).

[3] F. Matsukura, H. Ohno, A. Shen, and Y. Sugawara, Phys. Rev. B 57, R2037 (1998),

[4] H. Ohno, J. Magn. Magn. Mater, 200, 110 (1999).

[5] S. J. Potashnik, K. C. Ku, S. H. Chun, J. J. Berry, N. Samarth, and P. Schiffer, Appl. Phys. Lett. 79, 1495 (2001).

[6] K. W. Edmonds, K. Y. Wang, R. P. Campion, A. C. Neumann, C. T. Foxon, B. L. Gallagher, and P. C. Main, Appl. Phys. Lett. 81, 3010 (2002).

[7] M. J. Seong, S. H. Chun, H. M. Cheong, N. Samarth, and A. Mascarenhas, Phys. Rev. B 66, 033202 (2002),
[8] H. Asklund, L. Ilver, J. Kanski, and J. Sadowski, Phys. Rev. B 66, 115319 (2002).

[9] K. M. Yu, W. Walukiewicz, T. Wojtowicz, I. Kuryliszyn, X. Liu, Y. Sasaki, and J. K. Furdyna, Phys. Rev. B 65, 201303 (2002).

[10] K. M. Yu, W. Walukiewicz, T. Wojtowicz, W. L. Lim, X. Liu, Y. Sasaki, M. Dobrowolska, and J. K. Furdyna, Appl. Phys. Lett. 81, 844 (2002).

[11] S. J. Potashnik, K. C. Ku, R. Nahendiran, S. H. Chun, R. F. Wang, N. Samarth, and P. Schiffer, Phys. Rev. B 66, 012408 (2002).

[12] R. Moriya and H. Munekata, J. Appl. Phys. 93, 4603 (2002).

[13] R. R. dos Santos, L. E. Oliveira, and J. d'Albuquerque e Castro, J. Phys.: Cond. Matter, 14, 3751 (2002).

[14] R. R. dos Santos, J. d'Albuquerque e Castro, and L. E. Oliveira. J. Appl. Phys. 93, 1845 (2003). 
[15] M. van Schilfgaarde and O. N. Mryasov, Phys. Rev. B 63, 233205 (2001).

[16] J. L. Xu, M. van Schilfgaarde, and G. D. Samolyuk, Phys. Rev. Lett. 94, 097201 (2005).

[17] J. Kudrnovský, I. Turek, V. Drchal, F. Máca, P. Weinberger, and P. Bruno, Phys. Rev. B 69, 115208 (2004).

[18] J. Kudrnovský, V. Drchal, I. Turek, L. Berqvist, O. Eriksson, G. Bouzerar, L. Sandratskii, and P. Bruno, J. Phys.: Cond. Matter 16, S5571 (2004).

[19] L. Bergqvist, O. Eriksson, J. Kudrnovský, V. Drchal, P. Korzhavyi, and I. Turek, Phys. Rev. Lett. 93, 137202 (2004).

[20] K. Sato, H. Katayama-Yoshida, P. H. Dederichs, J. Superconductivity, 16, 31 (2003).

[21] K. Sato, W. Schweika, P. H. Dederichs, and H. KatayamaYoshida, Phys. Rev. B 70, 201202 (2004).

[22] L. M. Sandratskii and P. Bruno, Phys. Rev. B 66, 134435 (2002).

[23] L. M. Sandratskii and P. Bruno, Phys. Rev. B 67, 214402 (2003).

[24] L. M. Sandratskii and P. Bruno, J. Phys.: Cond. Matter 16, L523 (2004).

[25] D. Vanderbilt, Phys. Rev. B 41, 7892 (1990).

[26] G. Kresse and J. Hafner, Phys. Rev. B 47, R558 (1993).
[27] S. H. Wei, L. G. Ferreira, J. E. Bernard, and A. Zunger, Phys. Rev. B 42, 9622 (1990).

[28] A. J. R. da Silva, A. Fazzio, R. R. dos Santos, and L. E. Oliveira, Physica B 340-342, 874 (2003).

[29] A. J. R. da Silva, A. Fazzio, R. R. dos Santos, and L. E. Oliveira, J. Phys.: Cond. Matter bf16, 8243 (2004).

[30] A. J. R. da Silva, A. Fazzio, R. R. dos Santos, and L. E. Oliveira, Phys. Rev. B 72, 125208 (2005).

[31] P. Mahadevan and A. Zunger, Phys. Rev. B 69, 115211 (2004).

[32] P. Mahadevan, A. Zunger, and D. D. Sarma, Phys. Rev. Lett. 93, 177201 (2004).

[33] P. Mahadevan, J. M. Osorio-Guillén, and A. Zunger, Appl. Phys. Lett. 86, 172504 (2005).

[34] D. Stauffer and A. Aharony, Introduction to Percolation Theory, (Taylor \& Francis, London, 1992), 2nd Ed.

[35] V. F. Sapega, M. Moreno, M. Ramsteiner, L. Däweritz, and K. H. Ploog, Phys. Rev. Lett. 94, 137401 (2005).

[36] D. J. Priour, Jr., E. H. Hwang, and S. Das Sarma, Phys. Rev. Lett. 92, 117201 (2004).

[37] C. Timm and A. H. MacDonald, Phys. Rev. B 71, 155206 (2005). 